\title{
The Difference Splitting Scheme for Hyperbolic Systems with Variable Coefficients
}

\author{
Aloev R. D. ${ }^{1, *}$, Eshkuvatov Z. K. ${ }^{2}$, Khudoyberganov M. U. ${ }^{1}$, Nematova D. E. ${ }^{1}$ \\ ${ }^{1}$ Faculty of Mathematics, National University of Uzbekistan (NUUz), Tashkent, Uzbekistan \\ ${ }^{2}$ Faculty of Science and Technology, Universiti Sains Islam Malaysia (USIM), Negeri Sembilan, Malaysia
}

Copyright (C) 2019 by authors, all rights reserved. Authors agree that this article remains permanently open access under the terms of the Creative Commons Attribution License 4.0 International License

\begin{abstract}
In the paper, we propose a systematic approach to design and investigate the adequacy of the computational models for a mixed dissipative boundary value problem posed for the symmetric t-hyperbolic systems. We consider a two-dimensional linear hyperbolic system with variable coefficients and with the lower order term in dissipative boundary conditions. We construct the difference splitting scheme for the numerical calculation of stable solutions for this system. A discrete analogue of the Lyapunov's function is constructed for the numerical verification of stability of solutions for the considered problem. A priori estimate is obtained for the discrete analogue of the Lyapunov's function. This estimate allows us to assert the exponential stability of the numerical solution. A theorem on the exponential stability of the solution of the boundary value problem for linear hyperbolic system and on stability of difference splitting scheme in the Sobolev spaces was proved. These stability theorems give us the opportunity to prove the convergence of the numerical solution.
\end{abstract}

Keywords Difference Scheme, Lyapunov Function, Mixed Problem, Stability

\section{Introduction}

We consider the mixed dissipative boundary value problem for a two-dimensional linear hyperbolic system with variable coefficients and lower order term [1]. For this problem, we construct and investigate the difference splitting scheme in order to obtain stable solutions. A discrete analogue of the Lyapunov function is constructed and an a-priori estimate is obtained for it. The obtained a-priori estimate allows us to assert the exponential stability of the numerical solution.

It should be noted that numerous problems have been devoted to the solution of such problems (see [3]-[10] and references in them). Studying the stability of solutions for onedimensional hyperbolic systems is the subject of [2]. Authors of the paper investigate the stability of the solution by constructing the Lyapunov function and using a priori estimates for solution in various functional spaces. However, stability of the difference schemes, constructed in all these papers, was investigated using the technique of constructing dissipative energy integrals. The a-priori estimates are obtained in these papers. But they are not enough to get exponential stability of the numerical solution.

\section{Differential statement of the problem}

In the domain $G=\{(t, x, y): 0<t \leq T, 0<x<l,-\infty<$ $y<+\infty\}$, we considered a symmetric hyperbolic system in a special canonical form

$$
\frac{\partial \mathbf{v}}{\partial t}+\mathbf{K} \frac{\partial \mathbf{v}}{\partial x}+\mathbf{C} \frac{\partial \mathbf{v}}{\partial y}+\mathbf{M v}=\mathbf{0}
$$

with boundary conditions for $x=0$ :

$$
\mathbf{v}^{I}=\mathbf{s} \mathbf{v}^{I I}
$$

for $x=l$ :

$$
\mathbf{v}^{I I}=\mathbf{r} \mathbf{v}^{I}
$$

and with initial data at $t=0$

$$
\begin{array}{r}
v_{i}(0, x, y)=\varphi_{i}(x, y), i=1, \ldots, n, 0 \leq x \leq l, \\
-\infty \leq y \leq+\infty
\end{array}
$$

where $\mathbf{v}^{\mathbf{I}}=\left(v_{1}, v_{2}, \ldots, v_{m}\right)^{T}, \quad \mathbf{v}^{I I}=$ $\left(v_{m+1}, v_{m+2}, \ldots, v_{n}\right)^{T}, \mathbf{K}$ is a diagonal matrix, $\mathbf{C}$ is a positive definite matrix, $\mathbf{M}$ is a $n$-th order square real matrix

$$
\begin{aligned}
\mathbf{v} & =\left[\begin{array}{c}
\mathbf{v}^{I} \\
\mathbf{v}^{I I}
\end{array}\right], \mathbf{K}=\left(\begin{array}{cc}
K^{+} & 0 \\
0 & -K^{-}
\end{array}\right), \\
\mathbf{K}^{+} & =\left(\begin{array}{cccc}
k_{1} & 0 & \cdots & 0 \\
0 & k_{2} & \cdots & 0 \\
\vdots & \vdots & \ddots & \vdots \\
0 & \cdots & 0 & k_{m}
\end{array}\right), \\
\mathbf{K}^{-} & =\left(\begin{array}{cccc}
k_{m+1} & 0 & \cdots & 0 \\
0 & k_{m+2} & \cdots & 0 \\
\vdots & \vdots & \ddots & \vdots \\
0 & \cdots & 0 & k_{n}
\end{array}\right), k_{i}>0, i=1, \ldots, n ;
\end{aligned}
$$


$\mathbf{C}=\left\|c_{i j}(x)\right\|, \mathbf{M}=\left\|m_{i j}(x)\right\|, i, j=1, \ldots, n, 0 \leq x \leq l$, $\mathbf{s}$ is a matrix of order $(n-m) \times m, \mathbf{r}$ is a matrix of order $m \times(n-m)$. The initial functions are assumed to vanish for $|y|>\frac{1}{2} Y$. The similar problem is considered in [1].

The assumption of positive definiteness of the matrix $\mathbf{C}$ is not mandatory [1]. It is introduced to simplify the construction of the difference scheme. In fact, if the matrix $\mathbf{C}$ does not satisfy this assumption, then by introducing new coordinates $x^{\prime}, y^{\prime}, t^{\prime}$

$$
x^{\prime}=x, y^{\prime}=y+\omega t, t^{\prime}=t
$$

we can rewrite the equation in the form of

$$
\frac{\partial \mathbf{v}}{\partial t^{\prime}}+\mathbf{K} \frac{\partial \mathbf{v}}{\partial x^{\prime}}+(\mathbf{C}+\omega \mathbf{E}) \frac{\partial \mathbf{v}}{\partial y^{\prime}}+\mathbf{M} \mathbf{v}=0
$$

where the symmetric matrix $\mathbf{C}+\omega \mathbf{E}$ of the coefficients at $\frac{\partial \mathbf{v}}{\partial y^{\prime}}$ will be positively definite for a sufficiently large $\omega$. Here $\mathbf{E}$ is the identity matrix.

Suppose that the initial data $\varphi=\left(\varphi_{1}, \varphi_{2}, \ldots, \varphi_{n}\right)^{T} \in$ $W_{2}^{1}\left((0, l),(-\infty,+\infty), \mathbf{R}^{n}\right)$ satisfy the compatibility conditions:

$$
\begin{aligned}
& \varphi^{\mathbf{I}}=\mathbf{s} \varphi^{\mathbf{I I}}, x=0, t=0, \\
& \varphi^{\mathbf{I I}}=\mathbf{r} \varphi^{\mathbf{I}}, x=l, t=0 .
\end{aligned} .
$$

Here $W_{2}^{1}\left((0, l),(-\infty,+\infty), \mathbf{R}^{n}\right)$ is the Sobolev space.

Definition 2 (exponential stability [2]). The system (1) with boundary conditions (2)-(3) is said to be exponentially stable in the $\mathbf{L}^{2}-$ norm if there exists such $\nu>0$ and $c>0$ that for any initial condition $\varphi \in \mathbf{L}^{2}\left((0, l),(-\infty,+\infty), \mathbf{R}^{n}\right)$, the $\mathbf{L}^{2}-$ solution of the mixed problem (1)-(4) satisfies the inequality

$$
\begin{array}{r}
\|\mathbf{v}(t, \cdot)\|_{\mathbf{L}^{2}\left((0, l),(-\infty,+\infty) ; \mathbf{R}^{n}\right)} \leq \\
\leq c e^{-\nu t}\|\varphi\|_{\mathbf{L}^{2}\left((0, l),(-\infty,+\infty) ; \mathbf{R}^{n}\right)}, t \geq 0
\end{array}
$$

We construct the Lyapunov's function in the form:

$$
\begin{gathered}
L(t)=\int_{-\infty}^{+\infty} \int_{0}^{l}(\mu(x) \mathbf{v}, \mathbf{v}) d x d y= \\
=\int_{-\infty}^{+\infty} \int_{0}^{l}\left\{\sum_{i=1}^{m} \mu_{i} e^{-\nu x}\left[v_{i}(t, x, y)\right]^{2}+\right. \\
\left.+\sum_{i=m+1}^{n} \mu_{i} e^{\nu x}\left[v_{i}(t, x, y)\right]^{2}\right\} d x d y
\end{gathered}
$$

where $\mu_{i}>0, i=1, \ldots, n, \mu^{+}=\left(\mu_{1}, \ldots, \mu_{m}\right)^{T}$, $\mu^{-}=\left(\mu_{m+1}, \ldots, \mu_{n}\right)^{T}$,

$$
\mu(x)=\left(\begin{array}{cc}
e^{-\nu x} \mu^{+} & 0 \\
0 & e^{\nu x} \mu^{-}
\end{array}\right) .
$$

Theorem (exponential stability). The system (1) with the boundary conditions (2)-(3) is exponentially stable in the $\mathbf{L}^{2}$ -norm if there exists $\nu>0$ and $\mu_{i}>0, i=1, \ldots, n$ such that the following matrices

$$
\begin{gathered}
\left(\begin{array}{cc}
\mathbf{K}^{+}(l) & 0 \\
0 & \mathbf{K}^{-}(0)
\end{array}\right)\left(\begin{array}{cc}
e^{-\nu l} \mu^{+} & 0 \\
0 & \mu^{-}
\end{array}\right)-\left(\begin{array}{cc}
0 & \mathbf{r} \\
\mathbf{s} & 0
\end{array}\right) \times \\
\times\left(\begin{array}{cc}
\mathbf{K}^{+}(0) & 0 \\
0 & \mathbf{K}^{-}(l)
\end{array}\right)\left(\begin{array}{cc}
\mu^{+} & 0 \\
0 & e^{\nu l} \mu^{-}
\end{array}\right)\left(\begin{array}{ll}
0 & \mathbf{s} \\
\mathbf{r} & 0
\end{array}\right)
\end{gathered}
$$

and

$$
\begin{array}{r}
\nu|\mathbf{K}(x)| \mu(x)-\mathbf{K}^{\prime}(x) \mu(x)+\mu(x) \mathbf{M}^{T}(x) \mu(x)+ \\
+\mu(x) \mathbf{M}(x), x \in(0, l)
\end{array}
$$

are positive definite.

Proof. We calculate the derivative of the Lyapunov function: $L^{\prime}(t)=\int_{-\infty}^{+\infty} \int_{0}^{l} \partial_{t}(\mu(x) \mathbf{v}, \mathbf{v}) d x d y=$ $=\int_{-\infty}^{+\infty} \int_{0}^{l}\left(\mu(x) \partial_{t} \mathbf{v}, \mathbf{v}\right) d x d y+\int_{-\infty}^{+\infty} \int_{0}^{l}\left(\mu(x) \mathbf{v}, \partial_{t} \mathbf{v}\right) d x d y=$ $=\int_{-\infty}^{+\infty} \int_{0}^{l}\left(\mu(x)\left[-\mathbf{K}(x) \partial_{x} \mathbf{v}-\mathbf{C}(\mathbf{x}) \partial_{y} \mathbf{v}-\mathbf{M}(x) \mathbf{v}\right], \mathbf{v}\right) d x d y+$ $+\infty l$

$+\int_{-\infty}^{+\infty} \int_{0}^{l}\left(\mu(x) \mathbf{v},\left[-\mathbf{K}(x) \partial_{x} \mathbf{v}-\mathbf{C}(x) \partial_{y} \mathbf{v}-\mathbf{M}(\mathbf{x}) \mathbf{v}\right]\right) d x d y=$ $=-\int_{-\infty}^{+\infty} \int_{0}^{l}\left[\left(\mu(x) \mathbf{K}(x) \partial_{x} \mathbf{v}, \mathbf{v}\right)+\left(\mu(x) \mathbf{v}, \mathbf{K}(x) \partial_{x} \mathbf{v}\right)+\right.$ $\left.+\left(\mu(x) \mathbf{C}(x) \partial_{y} \mathbf{v}, \mathbf{v}\right)+\left(\mu(x) \mathbf{v}, \mathbf{C}(x) \partial_{y} \mathbf{v}\right)\right] d x d y-$ $+\infty l$

$-\int_{-\infty}^{+\infty} \int_{0}^{l}[(\mu(x) \mathbf{M}(\mathbf{x}) \mathbf{v}, \mathbf{v})+(\mu(x) \mathbf{v}, \mathbf{M}(\mathbf{x}) \mathbf{v})] d x d y$

Since

$\left(\mu(x) \mathbf{K}(\mathbf{x}) \partial_{x} \mathbf{v}, \mathbf{v}\right)=\left(\partial_{x}[\mu(x) \mathbf{K}(\mathbf{x}) \mathbf{v}], \mathbf{v}\right)-$ $-\left(\mu^{\prime}(x) \mathbf{K}(x) \mathbf{v}, \mathbf{v}\right)-\left(\mu(x) \mathbf{K}^{\prime}(x) \mathbf{v}, \mathbf{v}\right)$

and

$$
\mu^{\prime}(x) \mathbf{K}(x)=-\nu|\mathbf{K}(x)| \mu(x),
$$

we have the following identity:

$L^{\prime}(t)=-\int_{-\infty}^{+\infty} \int_{0}^{l}\left[\partial_{x}(\mathbf{K}(x) \mu(x) \mathbf{v}, \mathbf{v})+\partial_{y}(\mathbf{C}(x) \mu(x) \mathbf{v}, \mathbf{v})\right] d x d y-$

$-\int_{-\infty}^{+\infty} \int_{0}^{l}\left(\left[\nu|\mathbf{K}(x)| \mu(x)-\mathbf{K}^{\prime}(x) \mu(x)+\mathbf{M}^{T}(x) \mu(x)+\right.\right.$

$+\mu(x) \mathbf{M}(x)] \mathbf{v}, \mathbf{v}) d x d y=-\left.\int_{-\infty}^{+\infty}(\mathbf{K}(x) \mu(x) \mathbf{v}, \mathbf{v})\right|_{0} ^{l} d y-$

$-\left.\int_{0}^{l}(\mathbf{C}(x) \mu(x) \mathbf{v}, \mathbf{v})\right|_{-\infty} ^{+\infty} d x-\int_{-\infty}^{+\infty} \int_{0}^{l}([\nu|\mathbf{K}(x)| \mu(x)-$

$\left.\left.\mathbf{K}^{\prime}(x) \mu(x)+\mathbf{M}^{T}(x) \mu(x)+\mu(x) \mathbf{M}(x)\right] \mathbf{v}, \mathbf{v}\right) d x d y=$

$=-\left.\int_{-\infty}^{+\infty}(\mathbf{K}(x) \mu(x) \mathbf{v}, \mathbf{v})\right|_{0} ^{l} d y-\int_{-\infty}^{+\infty} \int_{0}^{l}([\nu|\mathbf{K}(x)| \mu(x)-$

$\left.\left.\mathbf{K}^{\prime}(x) \mu(x)+\mathbf{M}^{T}(x) \mu(x)+\mu(x) \mathbf{M}(x)\right] \mathbf{v}, \mathbf{v}\right) d x d y$.

Transform separately the each term of the obtained identity:

$1)-\left.(\mathbf{K}(x) \mu(x) \mathbf{v}, \mathbf{v})\right|_{0} ^{l}=-[(\mathbf{K}(l) \mu(l) \mathbf{v}(t, l, y), \mathbf{v}(t, l, y))-$

$(\mathbf{K}(0) \mu(0) \mathbf{v}(t, 0, y), \mathbf{v}(t, 0, y))]=$

$=-\left(\left(\begin{array}{cc}\mathbf{K}^{+}(l) & 0 \\ 0 & -\mathbf{K}^{-}(l)\end{array}\right)\left(\begin{array}{cc}e^{-\nu l} \mu^{+} & 0 \\ 0 & e^{\nu l} \mu^{-}\end{array}\right) \times\right.$ $\left.\times\left[\begin{array}{c}\mathbf{v}^{\mathrm{I}}(t, l, y) \\ \mathbf{v}^{\mathrm{II}}(t, l, y)\end{array}\right],\left[\begin{array}{c}\mathbf{v}^{\mathrm{I}}(t, l, y) \\ \mathbf{v}^{\mathrm{II}}(t, l, y)\end{array}\right]\right)+$ 


$$
\begin{aligned}
& +\left(\left(\begin{array}{cc}
\mathbf{K}^{+}(0) & 0 \\
0 & -\mathbf{K}^{-}(0)
\end{array}\right)\left(\begin{array}{cc}
\mu^{+} & 0 \\
0 & \mu^{-}
\end{array}\right) \times\right. \\
& \left.\times\left[\begin{array}{c}
\mathbf{v}^{\mathrm{I}}(t, 0, y) \\
\mathbf{v}^{\mathrm{II}}(t, 0, y)
\end{array}\right],\left[\begin{array}{c}
\mathbf{v}^{\mathrm{I}}(t, 0, y) \\
\mathbf{v}^{\mathrm{II}}(t, 0, y)
\end{array}\right]\right)= \\
& =-\left(\left(\begin{array}{cc}
\mathbf{K}^{+}(l) & 0 \\
0 & \mathbf{K}^{-}(0)
\end{array}\right)\left(\begin{array}{cc}
e^{-\nu l} \mu^{+} & 0 \\
0 & \mu^{-}
\end{array}\right) \times\right. \\
& \left.\times\left[\begin{array}{c}
\mathbf{v}^{\mathrm{I}}(t, l, y) \\
\mathbf{v}^{\mathrm{II}}(t, 0, y)
\end{array}\right],\left[\begin{array}{c}
\mathbf{v}^{\mathrm{I}}(t, l, y) \\
\mathbf{v}^{\mathrm{II}}(t, 0, y)
\end{array}\right]\right)+ \\
& +\left(\left(\begin{array}{cc}
\mathbf{K}^{+}(0) & 0 \\
0 & \mathbf{K}^{-}(l)
\end{array}\right)\left(\begin{array}{cc}
\mu^{+} & 0 \\
0 & e^{\nu l} \mu^{-}
\end{array}\right) \times\right. \\
& \left.\times\left[\begin{array}{c}
\mathbf{v}^{\mathrm{I}}(t, 0, y) \\
\mathbf{v}^{\mathrm{II}}(t, l, y)
\end{array}\right],\left[\begin{array}{c}
\mathbf{v}^{\mathrm{I}}(t, 0, y) \\
\mathbf{v}^{\mathrm{II}}(t, l, y)
\end{array}\right]\right)= \\
& =-\left(\left(\begin{array}{cc}
\mathbf{K}^{+}(l) & 0 \\
0 & \mathbf{K}^{-}(0)
\end{array}\right)\left(\begin{array}{cc}
e^{-\nu l} \mu^{+} & 0 \\
0 & \mu^{-}
\end{array}\right) \times\right. \\
& \left.\times\left[\begin{array}{c}
\mathbf{v}^{\mathrm{I}}(t, l, y) \\
\mathbf{v}^{\mathrm{II}}(t, 0, y)
\end{array}\right],\left[\begin{array}{c}
\mathbf{v}^{\mathrm{I}}(t, l, y) \\
\mathbf{v}^{\mathrm{II}}(t, 0, y)
\end{array}\right]\right)+ \\
& +\left(\left(\begin{array}{cc}
\mathbf{K}^{+}(0) & 0 \\
0 & \mathbf{K}^{-}(l)
\end{array}\right)\left(\begin{array}{cc}
\mu^{+} & 0 \\
0 & e^{\nu l} \mu^{-}
\end{array}\right) \times\right. \\
& \times\left(\begin{array}{ll}
0 & \mathbf{s} \\
\mathbf{r} & 0
\end{array}\right)\left[\begin{array}{c}
\mathbf{v}^{\mathrm{I}}(t, l, y) \\
\mathbf{v}^{\mathrm{II}}(t, 0, y)
\end{array}\right],\left(\begin{array}{ll}
0 & \mathbf{s} \\
\mathbf{r} & 0
\end{array}\right) \times \\
& \left.\times\left[\begin{array}{c}
\mathbf{v}^{\mathrm{I}}(t, l, y) \\
\mathbf{v}^{\mathrm{II}}(t, 0, y)
\end{array}\right]\right)=-\left(\left(\begin{array}{cc}
\mathbf{K}^{+}(l) & 0 \\
0 & \mathbf{K}^{-}(0)
\end{array}\right) \times\right. \\
& \times\left(\begin{array}{cc}
e^{-\nu l} \mu^{+} & 0 \\
0 & \mu^{-}
\end{array}\right)\left[\begin{array}{c}
\mathbf{v}^{\mathrm{I}}(t, l, y) \\
\mathbf{v}^{\mathrm{II}}(t, 0, y)
\end{array}\right] \text {, } \\
& \left.,\left[\begin{array}{c}
\mathbf{v}^{\mathrm{I}}(t, l, y) \\
\mathbf{v}^{\mathrm{II}}(t, 0, y)
\end{array}\right]\right)+\left(\left(\begin{array}{cc}
0 & \mathbf{r} \\
\mathbf{s} & 0
\end{array}\right) \times\right. \\
& \times\left(\begin{array}{cc}
\mathbf{K}^{+}(0) & 0 \\
0 & \mathbf{K}^{-}(l)
\end{array}\right)\left(\begin{array}{cc}
\mu^{+} & 0 \\
0 & e^{\nu l} \mu^{-}
\end{array}\right)\left(\begin{array}{ll}
0 & \mathbf{s} \\
\mathbf{r} & 0
\end{array}\right) \times \\
& \left.\times\left[\begin{array}{c}
\mathbf{v}^{\mathrm{I}}(t, l, y) \\
\mathbf{v}^{\mathrm{II}}(t, 0, y)
\end{array}\right],\left[\begin{array}{c}
\mathbf{v}^{\mathrm{I}}(t, l, y) \\
\mathbf{v}^{\mathrm{II}}(t, 0, y)
\end{array}\right]\right)<0 .
\end{aligned}
$$

According to (9) we have

$2)-\int_{0}^{l}\left(\left[\nu|\mathbf{K}(x)| \mu(x)-\mathbf{K}^{\prime}(x) \mu+\right.\right.$

$$
\left.\left.+\mathbf{M}^{T}(x) \mu(x)+\mu(x) \mathbf{M}(x)\right] \mathbf{v}, \mathbf{v}\right) d x<0 \text {. }
$$

Taking into account these transformations, we obtain $L^{\prime}(t)=-\left.\int_{-\infty}^{+\infty}(\mathbf{K}(x) \mu(x) \mathbf{v}, \mathbf{v})\right|_{0} ^{l} d y-\int_{-\infty}^{+\infty} \int_{0}^{l}([\nu|\mathbf{K}(x)| \times$ $\times \mu(x)-\mathbf{K}^{\prime}(x) \mu(x)+\mathbf{M}^{T}(x) \mu(x)+$

$+\mu(x) \mathbf{M}(x)] \mathbf{v}, \mathbf{v}) d x d y<0$.

Since the matrices (9) and $\nu|\mathbf{K}(x)| \mu(x)$ are positive definite for any $x$,we get following inequality $\left(\left[\nu|\mathbf{K}(x)| \mu(x)-\mathbf{K}^{\prime}(x) \mu(x)+\mathbf{M}^{T}(x) \mu(x)+\right.\right.$

$+\mu(x) \mathbf{M}(x)] \mathbf{v}, \mathbf{v})>\nu(|\mathbf{K}(x)| \mu(x) \mathbf{v}, \mathbf{v})>\nu \alpha(\mu(x) \mathbf{v}, \mathbf{v})$,

$$
\alpha=\nu \min _{\substack{1 \leq i \leq n \\ 0 \leq x \leq l}} k_{i}(x)
$$

for any vector $\mathbf{v} \in \mathbf{R}^{n}$. Then we have

$L^{\prime}(t)<-\nu \alpha \int_{-\infty}^{+\infty} \int_{0}^{l}(\mu(x) \mathbf{v}, \mathbf{v}) d x d y=-\eta L(t), \quad \eta=\nu \alpha$

Hence,

$$
L(t) \leq e^{-\eta t} L(0), \quad t>0 .
$$

However, since there is such a constant $\gamma>0$ such that $\frac{1}{\gamma}\|\mathbf{v}(t, \cdot)\|_{L^{2}\left((0, l),(-\infty,+\infty), R^{n}\right)}^{2} \leq L(t) \leq$ $\leq \gamma\|\mathbf{v}(t, \cdot)\|_{L^{2}\left((0, l),(-\infty,+\infty), R^{n}\right)}^{2}$, $\|\mathbf{v}(t, \cdot)\|_{L^{2}\left((0, l),(-\infty,+\infty), R^{n}\right)}^{2}=\int_{-\infty}^{+\infty} \int_{0}^{l}(\mathbf{v}, \mathbf{v}) d x d y$

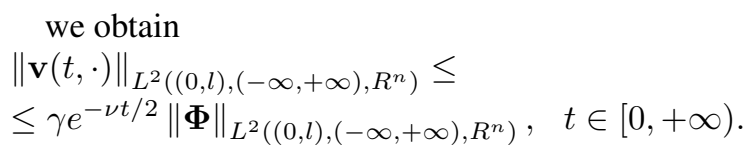

\section{The difference scheme}

In the domain $G$, construct the difference grid

$G_{h}=\left\{\left(t^{\kappa}, x_{j}, y_{q}\right): 0 \leq t^{\kappa} \leq T, 0 \leq x_{j} \leq l,-\infty<\right.$ $\left.y_{q}<+\infty\right\}$ where $t^{\kappa}=\kappa \Delta t, \kappa=0, \ldots, N ; N \Delta t=T$,

$$
\begin{aligned}
& x_{j}=\left(j+\frac{1}{2}\right) \Delta x ; J \Delta x=l ; j=0, \ldots, J-1 ; \\
& y_{q}=\left(q+\frac{1}{2}\right) \Delta y ; q=-\infty, \ldots,+\infty ;
\end{aligned}
$$

and denote the values of the numerical solution at the nodal points by

$$
\mathbf{v}_{j q}^{\kappa}=\frac{1}{\Delta x} \frac{1}{\Delta y} \int_{y_{q-\frac{1}{2}}}^{y_{q+\frac{1}{2}}} \int_{x_{j-\frac{1}{2}}}^{x_{j+\frac{1}{2}}} \mathbf{v}\left(t^{\kappa}, x, y\right) d x d y, \quad j=0, \ldots, J-1 .
$$

For the numerical solution of the mixed problem (1)-(4), we suggest a difference splitting scheme

$$
\begin{gathered}
{\left[\begin{array}{c}
\left(\mathbf{w}^{\mathrm{I}}\right)_{j q}^{\kappa} \\
\left(\mathbf{w}^{\mathrm{II}}\right)_{j q}^{\kappa}
\end{array}\right]=\left[\begin{array}{c}
\left(\mathbf{v}^{\mathrm{I}}\right)_{j q}^{\kappa} \\
\left(\mathbf{v}^{\mathrm{II}}\right)_{j q}^{\kappa}
\end{array}\right]-\frac{\Delta t}{\Delta x} \times} \\
\times\left[\begin{array}{cc}
\mathbf{K}_{j-1}^{+} & 0 \\
0 & \mathbf{K}_{j+1}^{-}
\end{array}\right]\left[\begin{array}{c}
\left(\mathbf{v}^{\mathrm{I}}\right)_{j q}^{\kappa}-\left(\mathbf{v}^{\mathrm{I}}\right)_{j-1, q}^{\kappa} \\
\left(\mathbf{v}^{\mathrm{II}}\right)_{j q}^{\kappa^{k}}-\left(\mathbf{v}^{\mathrm{II}}\right)_{j+1, q}^{\kappa}
\end{array}\right], \\
j=0, \ldots, J-1 ; \kappa=0, \ldots, N-1 ; \\
q=-\infty, \ldots,+\infty ; \\
\mathbf{u}_{j q}^{\kappa}=\mathbf{w}_{j q}^{\kappa}-\frac{\Delta t}{\Delta y} \mathbf{C}_{j}\left[\mathbf{w}_{j q}^{\kappa}-\mathbf{w}_{j q-1}^{\kappa}\right] \\
j=0, \ldots, J-1 ; \kappa=0, \ldots, N-1 ; \\
q=-\infty, \ldots,+\infty ; \\
\mathbf{v}_{j q}^{\kappa+1}=\mathbf{u}_{j q}^{\kappa}-\Delta t \mathbf{M}_{j} \mathbf{u}_{j q}^{\kappa}, \quad j=0, \ldots, J-1 ;
\end{gathered}
$$

The initial conditions (4) are approximated as follows:

$$
\begin{aligned}
\mathbf{v}_{j q}^{0} & =\frac{1}{\Delta x} \frac{1}{\Delta y} \int_{y_{q-\frac{1}{2}}}^{y_{q+\frac{1}{2}}} \int_{x_{j-\frac{1}{2}}}^{x_{j+\frac{1}{2}}} \boldsymbol{\Phi}(x, y) d x d y, \\
j & =0, \ldots, J-1 ; q=-\infty, \ldots+\infty
\end{aligned}
$$

The boundary conditions are approximated in this way:

$$
\left(\begin{array}{c}
\left(\mathbf{v}^{\mathrm{I}}\right)_{-1 q}^{\kappa+1} \\
\left(\mathbf{v}^{\mathrm{II}}\right)_{J q}^{\kappa+1}
\end{array}\right)=\left(\begin{array}{cc}
0 & \mathbf{s} \\
\mathbf{r} & 0
\end{array}\right)\left(\begin{array}{c}
\left(\mathbf{v}^{\mathrm{I}}\right)_{J-1, q}^{\kappa+1} \\
\left(\mathbf{v}^{\mathrm{II}}\right)_{0 q}^{\kappa+1}
\end{array}\right)
$$

Suppose that the condition of the CFL

$$
\frac{\Delta t}{\Delta x} \max _{\substack{1 \leq i \leq n \\ 0 \leq j \leq J-1}}\left|k_{i, j}\right| \leq 1, \quad \frac{\Delta t}{\Delta y} \max _{\substack{1 \leq i \leq n \\ 0 \leq j \leq J-1}}\left|\lambda_{i}\left(\mathbf{C}_{j}\right)\right| \leq 1
$$


holds. Here $\lambda_{i}\left(\mathbf{C}_{j}\right)$ are eigenvalues of the matrix $\mathbf{C}_{j}$.

Now we study the question on the exponential stability of the solution of the difference problem (10)-(14).

Definition 2. The difference scheme (10)-(12) with a difference boundary condition (14) exponentially stable if there exist constants $\eta>0$ and $c>0$ such that for any initial condition $\mathbf{v}_{j q}^{0} \in L^{2}\left(\left(x_{j-\frac{1}{2}}, x_{j+\frac{1}{2}}\right),\left(y_{q-\frac{1}{2}}, y_{q+\frac{1}{2}}\right), \mathbf{R}^{n}\right)$, the solution of the difference boundary value problem (10)-(14) satisfies the equality

$$
\begin{aligned}
& \Delta x \Delta y \sum_{q=-\infty}^{+\infty} \sum_{j=0}^{J-1}\left(\mathbf{v}_{j q}^{\kappa}, \mathbf{v}_{j q}^{\kappa}\right) \leq c e^{-\eta t_{\kappa}} \times \\
& \times \Delta x \Delta y \sum_{q=-\infty}^{+\infty} \sum_{j=0}^{J-1}\left(\mathbf{v}_{j q}^{0}, \mathbf{v}_{j q}^{0}\right), \kappa=1, \ldots, N .
\end{aligned}
$$

Here, we formally write out the sums of an infinite number of terms. However this is done only formally, since only a finite number of them are nonzero. It is due to the fact that the difference solution is non-zero only at a finite number of points.

Consider the difference boundary-value problem (10) - (14) with the stationary solution

$\mathbf{v}_{j q}^{\kappa}=0, \kappa=0, \ldots, N-1 ; j=0, \ldots, J-1 ;$

$q=-\infty, \ldots,+\infty$.

In order to prove the stability of the difference boundaryvalue problem (10) - (14), we propose the following function as the discrete Lyapunov function

$$
\begin{array}{r}
L\left(\mathbf{v}^{\kappa}\right)=L^{\kappa}=\Delta x \Delta y \sum_{q=-\infty}^{+\infty} \sum_{j=0}^{J-1}\left(\mathbf{v}_{j q}^{\kappa}, \mu_{j} \mathbf{v}_{j q}^{\kappa}\right), \\
\mu_{j}=\mu\left(x_{j}\right), j=1, \ldots, J-1
\end{array}
$$

$$
\text { Here } \mu_{j}=\left(\begin{array}{cc}
e^{-\nu x_{j}} \mu^{+} & 0 \\
0 & e^{\nu x_{j}} \mu^{-}
\end{array}\right) \text {. }
$$

Theorem 2. Let $T>0$ and the discrete Lyapunov function be defined by (15). If the condition of CFL $\frac{\Delta t}{\Delta x} \max _{\substack{1 \leq i \leq n \\ 0<j<J-1}}\left|k_{i, j}\right| \leq$ 1, $\frac{\Delta t}{\Delta y} \max _{\substack{1 \leq i \leq n \\ 0<j \leq J-1}}\left|\lambda_{i}\left(\mathbf{C}_{j}\right)\right| \leq 1$, holds, and there exist real numbers $\nu>0$ and $\mu_{i}>0, i=1, \ldots, n$ such that $0<\eta \equiv \nu \alpha e^{-\nu \Delta x}-\beta<1$, where $\alpha=\min _{\substack{1 \leq i \leq n \\ 0 \leq j \leq J-1}}\left|k_{i, j}\right|$, $\beta=\max _{\substack{1 \leq i \leq n \\ 0 \leq j \leq J-1}}\left|k_{i, j}^{\prime}\right|, \mathbf{M}_{j}^{T} \mu_{j}+\mu_{j} \mathbf{M}_{j}-\Delta t \mathbf{M}_{j}^{T} \mu_{j} \mathbf{M}_{j}, j=$ $0, \ldots, J-1$ are non-negative definite matrices and $\left[\begin{array}{cc}\mu^{+} e^{-\nu x_{J}} \mathbf{K}_{J-1}^{+} & 0 \\ 0 & \mu^{-} e^{\nu x_{-1}} \mathbf{K}_{0}^{-}\end{array}\right]-\left(\begin{array}{cc}0 & \mathbf{r} \\ \mathbf{s} & 0\end{array}\right) \times$
$\times\left[\begin{array}{cc}\mu^{+} e^{-\nu x_{0}} \mathbf{K}_{-1}^{+} & 0 \\ 0 & \mu^{-} e^{\nu x_{J-1}} \mathbf{K}_{J}^{-}\end{array}\right]\left(\begin{array}{cc}0 & \mathbf{s} \\ \mathbf{r} & 0\end{array}\right)$ is a positive definite matrix, then the numerical solution $\mathbf{v}_{j q}^{\kappa}$ of the difference boundary value problem (10)-(14) converges to the stationary solution $\mathbf{v}_{j q}^{*}=0$ for the norm $\mathbf{L}^{2}$.

Proof. Using the Lyapunov discrete function, we calculate the derivative of the Lyapunov function (15) as follows $\frac{L\left(\mathbf{v}^{\kappa+1}\right)-L\left(\mathbf{v}^{\kappa}\right)}{\Delta t}=\frac{L\left(\mathbf{v}^{\kappa+1}\right)-L\left(\mathbf{u}^{\kappa}\right)}{\Delta t}+\frac{L\left(\mathbf{u}^{\kappa}\right)-L\left(\mathbf{w}^{\kappa}\right)}{\Delta t}+$
$+\frac{L\left(\mathbf{w}^{\kappa}\right)-L\left(\mathbf{v}^{\kappa}\right)}{\Delta t}$

$$
\begin{aligned}
& \text { where } L\left(\mathbf{v}^{\kappa}\right)=\Delta x \Delta y \sum_{q=-\infty}^{+\infty} \sum_{j=0}^{J-1}\left(\mathbf{v}_{j q}^{\kappa}, \mu_{j} \mathbf{v}_{j q}^{\kappa}\right), L\left(\mathbf{w}^{\kappa}\right)= \\
& =\Delta x \Delta y \sum_{q=-\infty}^{+\infty} \sum_{j=0}^{J-1}\left(\mathbf{w}_{j q}^{\kappa}, \mu_{j} \mathbf{w}_{j q}^{\kappa}\right), \\
& L\left(\mathbf{u}^{\kappa}\right)=\Delta x \Delta y \sum_{q=-\infty}^{+\infty} \sum_{j=0}^{J-1}\left(\mathbf{u}_{j q}^{\kappa}, \mu_{j} \mathbf{u}_{j q}^{\kappa}\right), \kappa=0, \ldots, N .
\end{aligned}
$$

Now we prove that this quadratic form is negatively defined. For this, it suffices to show that all three quadratic forms on the right-hand side of the previous equation are negatively defined:

$$
\begin{aligned}
& \frac{L\left(\mathbf{v}^{\kappa+1}\right)-L\left(\mathbf{u}^{\kappa}\right)}{\Delta t}=\frac{\Delta x \Delta y}{\Delta t} \times \\
\times & \sum_{q=-\infty}^{+\infty} \sum_{j=0}^{J-1}\left[\left(\mathbf{v}_{j q}^{\kappa+1}, \mu_{j} \mathbf{v}_{j q}^{\kappa+1}\right)-\left(\mathbf{u}_{j q}^{\kappa}, \mu_{j} \mathbf{u}_{j q}^{\kappa}\right)\right], \\
& \frac{L\left(\mathbf{u}^{\kappa}\right)-L\left(\mathbf{w}^{\kappa}\right)}{\Delta t}=\frac{\Delta x \Delta y}{\Delta t} \times \\
\times & \sum_{q=-\infty} \sum_{j=0}^{J-1}\left[\left(\mathbf{u}_{j q}^{\kappa}, \mu_{j} \mathbf{u}_{j q}^{\kappa}\right)-\left(\mathbf{w}_{j q}^{\kappa}, \mu_{j} \mathbf{w}_{j q}^{\kappa}\right)\right], \\
& \frac{L\left(\mathbf{w}^{\kappa}\right)-L\left(\mathbf{v}^{\kappa}\right)}{\Delta t}=\frac{\Delta x \Delta y}{\Delta t} \times \\
\times & \sum_{q=-\infty}^{+\infty} \sum_{j=0}^{J-1}\left[\left(\mathbf{w}_{j q}^{\kappa}, \mu_{j} \mathbf{w}_{j q}^{\kappa}\right)-\left(\mathbf{v}_{j q}^{\kappa}, \mu_{j} \mathbf{v}_{j q}^{\kappa}\right)\right] .
\end{aligned}
$$

Lemma 1. Let the conditions of Theorem 2 be satisfied. Then the quadratic form

$$
\frac{L\left(\mathbf{v}^{\kappa+1}\right)-L\left(u^{\kappa}\right)}{\Delta t} \leq 0
$$

is not positively defined.

Proof. Using the difference scheme (12), calculate the quadratic form $\frac{L\left(\mathbf{v}^{\kappa+1}\right)-L\left(u^{\kappa}\right)}{\Delta t}$.

$$
\begin{aligned}
& \frac{L\left(\mathbf{v}^{\kappa+1}\right)-L\left(\mathbf{u}^{\kappa}\right)}{\Delta t}=\frac{\Delta x \Delta y}{\Delta t} \sum_{q=-\infty}^{+\infty} \sum_{j=0}^{J-1}\left[\left(\left[\mathbf{u}_{j q}^{\kappa}-\Delta t \mathbf{M}_{\mathbf{j}} \mathbf{u}_{j q}^{\kappa}\right],\right.\right. \\
& \left.\left.\mu_{j}\left[\mathbf{u}_{j q}^{\kappa}-\Delta t \mathbf{M}_{\mathbf{j}} \mathbf{u}_{j q}^{\kappa}\right]\right)-\left(\mathbf{u}_{j q}^{\kappa}, \mu_{j} \mathbf{u}_{j q}^{\kappa}\right)\right]=\frac{\Delta x \Delta y}{\Delta t} \times \\
& \times \sum_{q=-\infty}^{+\infty} \sum_{j=0}^{J-1}\left[\left(\mathbf{u}_{j q}^{\kappa}, \mu_{j} \mathbf{u}_{j q}^{\kappa}\right)-\left(\mathbf{u}_{j q}^{\kappa}, \mu_{j} \mathbf{u}_{j q}^{\kappa}\right)\right]-\Delta x \Delta y \times \\
& \times \sum_{q=-\infty}^{+\infty} \sum_{j=0}^{J-1}\left[\left(\mathbf{M}_{\mathbf{j}} \mathbf{u}_{j q}^{\kappa}, \mu_{j} \mathbf{u}_{j q}^{\kappa}\right)+\left(\mathbf{u}_{j q}^{\kappa}, \mu_{j} \mathbf{M}_{\mathbf{j}} \mathbf{u}_{j q}^{\kappa}\right)-\right. \\
& \left.-\Delta t\left(\mathbf{M}_{\mathbf{j}} \mathbf{u}_{j q}^{\kappa}, \mu_{j} \mathbf{M}_{\mathbf{j}} \mathbf{u}_{j q}^{\kappa}\right)\right]=-\Delta x \Delta y \times \\
& \times \sum_{q=-\infty}^{+\infty} \sum_{j=0}^{J-1}\left[\left(\mathbf{u}_{j q}^{\kappa}, \mathbf{M}_{j}^{T} \mu_{j} \mathbf{u}_{j q}^{\kappa}\right)+\left(\mathbf{u}_{j q}^{\kappa}, \mu_{j} \mathbf{M}_{\mathbf{j}} \mathbf{u}_{j q}^{\kappa}\right)-\right. \\
& \left.-\Delta t\left(\mathbf{u}_{j q}^{\kappa}, \mathbf{M}_{j}^{T} \mu_{j} \mathbf{M}_{\mathbf{j}} \mathbf{u}_{j q}^{\kappa}\right)\right]=-\Delta x \Delta y \times \\
& \times \sum_{q=-\infty}^{+\infty} \sum_{j=0}^{J-1}\left(\mathbf{u}_{j q}^{\kappa},\left[\mathbf{M}_{j}^{T} \mu_{j}+\mu_{j} \mathbf{M}_{j}-\Delta t \mathbf{M}_{j}^{T} \mu_{j} \mathbf{M}_{j}\right] \mathbf{u}_{j q}^{\kappa}\right) .
\end{aligned}
$$

By virtue of Theorem 2. $\quad \mathbf{M}_{j}^{T} \mu_{j}+\mu_{j} \mathbf{M}_{j}-$ $\Delta t \mathbf{M}_{j}^{T} \mu_{j} \mathbf{M}_{j}, j=0, \ldots, J-1$ are nonnegative definite matrix. Hence,

$$
\frac{L\left(\mathbf{v}^{\kappa+1}\right)-L\left(\mathbf{u}^{\kappa}\right)}{\Delta t} \leq 0 .
$$

Lemma 1 is proved.

Lemma 2. Let the conditions of Theorem 2 be satisfied. Then the quadratic form

$$
\frac{L\left(\mathbf{u}^{\kappa}\right)-L\left(\mathbf{w}^{\kappa}\right)}{\Delta t} \leq 0
$$


is not positively defined.

Proof. Introduce the notation $\mathbf{O}_{j}=\frac{\Delta t}{\Delta y} \mathbf{C}_{j}$. Using the difference scheme (11), calculate the quadratic form $\frac{L\left(\mathbf{u}^{\kappa}\right)-L\left(\mathbf{w}^{\kappa}\right)}{\Delta t}$.

$\frac{L\left(\mathbf{u}^{\kappa}\right)-L\left(\mathbf{w}^{\kappa}\right)}{\Delta t}=\frac{\Delta x \Delta y}{\Delta t} \sum_{q=-\infty}^{+\infty} \sum_{j=0}^{J-1}\left[\left(\mathbf{u}_{j q}^{\kappa}, \mu_{j} \mathbf{u}_{j q}^{\kappa}\right)-\left(\mathbf{w}_{j q}^{\kappa}, \mu_{j} \mathbf{w}_{j q}^{\kappa}\right)\right.$

$\frac{\Delta x \Delta y}{\Delta t} \sum_{q=-\infty}^{+\infty} \sum_{j=0}^{J-1}\left[\left(\left\{\mathbf{w}_{j q}^{\kappa}-\mathbf{O}_{j}\left[\mathbf{w}_{j q}^{\kappa}-\mathbf{w}_{j q-1}^{\kappa}\right]\right\}, \mu_{j}\left\{\mathbf{w}_{j q}^{\kappa}-\right.\right.\right.$

$\left.\left.\left.-\mathbf{O}_{j}\left[\mathbf{w}_{j q}^{\kappa}-\mathbf{w}_{j q-1}^{\kappa}\right]\right\}\right)-\left(\mathbf{w}_{j q}^{\kappa}, \mu_{j} \mathbf{w}_{j q}^{\kappa}\right)\right] \frac{\Delta x \Delta y}{\Delta t} \sum_{q=-\infty}^{+\infty} \times$

$\times \sum_{j=0}^{J-1}\left\{\left(\mathbf{w}_{j q}^{\kappa}, \mu_{j} \mathbf{w}_{j q}^{\kappa}\right)-\left(\mathbf{w}_{j q}^{\kappa}, \mu_{j} \mathbf{w}_{j q}^{\kappa}\right)-2\left(\mu_{j} \mathbf{w}_{j q}^{\kappa}, \mathbf{O}_{j} \times\right.\right.$

$\left.\left.\times\left[\mathbf{w}_{j q}^{\kappa}-\mathbf{w}_{j q-1}^{\kappa}\right]\right)\right\}+\frac{\Delta x \Delta y}{\Delta t} \sum_{q=-\infty}^{+\infty} \sum_{j=0}^{J-1}\left(\mu_{j} \mathbf{O}_{j}\left[\mathbf{w}_{j q}^{\kappa}-\mathbf{w}_{j q-1}^{\kappa}\right]\right.$,

$\left.\mathbf{O}_{j}\left[\mathbf{w}_{j q}^{\kappa}-\mathbf{w}_{j q-1}^{\kappa}\right]\right)=\frac{\Delta x \Delta y}{\Delta t} \sum_{q=-\infty}^{+\infty} \sum_{j=0}^{J-1}\left\{\left(\mu_{j} \mathbf{O}_{j}\left[\mathbf{w}_{j q}^{\kappa}-\mathbf{w}_{j q-1}^{\kappa}\right]\right.\right.$,

$\left.\left.\mathbf{O}_{j}\left[\mathbf{w}_{j q}^{\kappa}-\mathbf{w}_{j q-1}^{\kappa}\right]\right)-2\left(\mu_{j} \mathbf{w}_{j q}^{\kappa}, \mathbf{O}_{j}\left[\mathbf{w}_{j q}^{\kappa}-\mathbf{w}_{j q-1}^{\kappa}\right]\right)\right\}=$

$\frac{\Delta x \Delta y}{\Delta t} \sum_{q=-\infty}^{+\infty} \sum_{j=0}^{J-1}\left\{\left(\mu_{j} \mathbf{O}_{j} \mathbf{w}_{j q}^{\kappa}, \mathbf{O}_{j} \mathbf{w}_{j q}^{\kappa}\right)-2\left(\mu_{j} \mathbf{O}_{j} \mathbf{w}_{j q}^{\kappa}\right.\right.$,

$\left.\left.\mathbf{O}_{j} \mathbf{w}_{j q-1}^{\kappa}\right)+\left(\mu_{j} \mathbf{O}_{j} \mathbf{w}_{j q-1}^{\kappa}, \mathbf{O}_{j} \mathbf{w}_{j q-1}^{\kappa}\right)\right\}+$

$+\frac{\Delta x \Delta y}{\Delta t} \sum_{q=-\infty}^{+\infty} \sum_{j=0}^{J-1}\left\{-2\left(\mu_{j} \mathbf{w}_{j q}^{\kappa}, \mathbf{O}_{j} \mathbf{w}_{j q}^{\kappa}\right)+2\left(\mu_{j} \mathbf{w}_{j q}^{\kappa}\right.\right.$,

$\left.\left.\mathbf{O}_{j} \mathbf{w}_{j q-1}^{\kappa}\right)\right\}=\frac{\Delta x \Delta y}{\Delta t} \sum_{q=-\infty}^{+\infty} \sum_{j=0}^{J-1}\left\{\left(\mu_{j} \mathbf{O}_{j} \mathbf{w}_{j q}^{\kappa}, \mathbf{O}_{j} \mathbf{w}_{j q}^{\kappa}\right)+\right.$

$+2\left(\mu_{j}\left[\mathbf{E}-\mathbf{O}_{j}\right] \mathbf{w}_{j q}^{\kappa}, \mathbf{O}_{j} \mathbf{w}_{j q-1}^{\kappa}\right)+\left(\mu_{j} \mathbf{O}_{j} \mathbf{w}_{j q-1}^{\kappa}\right.$,

$\left.\left.\mathbf{O}_{j} \mathbf{w}_{j q-1}^{\kappa}\right)-2\left(\mu_{j} \mathbf{w}_{j q}^{\kappa}, \mathbf{O}_{j} \mathbf{w}_{j q}^{\kappa}\right)\right\}$.

Here $\mathbf{E}$ is the unit matrix. By virtue of the condition of the $\mathrm{CFL}$, the matrix $\left(\mathbf{E}-\mathbf{O}_{j}\right) \geq 0$. Suppose that the matrix $\left(\mathbf{E}-\mathbf{O}_{\mathbf{j}}\right) \mu_{j} \mathbf{O}_{j}$ is a positive definite matrix. Then we obtain

$2\left(\mu_{j}\left[\mathbf{E}-\mathbf{O}_{j}\right] \mathbf{w}_{j q}^{\kappa}, \mathbf{O}_{j} \mathbf{w}_{j q-1}^{\kappa}\right) \leq$

$\leq\left(\mu_{j}\left[\mathbf{E}-\mathbf{O}_{j}\right] \mathbf{w}_{j q}^{\kappa}, \mathbf{O}_{j} \mathbf{w}_{j q}^{\kappa}\right)+$

$+\left(\mu_{j}\left[\mathbf{E}-\mathbf{O}_{j}\right] \mathbf{w}_{j q-1}^{\kappa}, \mathbf{O}_{j} \mathbf{w}_{j q-1}^{\kappa}\right)=$

$\left(\mu_{j} \mathbf{w}_{j q}^{\kappa}, \mathbf{O}_{j} \mathbf{w}_{j q}^{\kappa}\right)-\left(\mu_{j} \mathbf{O}_{j} \mathbf{w}_{j q}^{\kappa}, \mathbf{O}_{j} \mathbf{w}_{j q}^{\kappa}\right)+$

$+\left(\mu_{j} \mathbf{w}_{j q-1}^{\kappa}, \mathbf{O}_{j} \mathbf{w}_{j q-1}^{\kappa}\right)-\left(\mu_{j} \mathbf{O}_{j} \mathbf{w}_{j q-1}^{\kappa}, \mathbf{O}_{j} \mathbf{w}_{j q-1}^{\kappa}\right)$.

Taking into account this inequality, we have

$\frac{L\left(\mathbf{u}^{\kappa}\right)-L\left(\mathbf{w}^{\kappa}\right)}{\Delta t}=$

$\frac{\Delta x \Delta y}{\Delta t} \sum_{q=-\infty}^{+\infty} \sum_{j=0}^{J-1}\left\{\begin{array}{l}\left(\mu_{j} \mathbf{O}_{j} \mathbf{w}_{j q}^{\kappa}, \mathbf{O}_{j} \mathbf{w}_{j q}^{\kappa}\right)+ \\ +2\left(\mu_{j}\left[\mathbf{E}-\mathbf{O}_{j}\right] \mathbf{w}_{j q}^{\kappa}, \mathbf{O}_{j} \mathbf{w}_{j q-1}^{\kappa}\right)+ \\ +\left(\mu_{j} \mathbf{O}_{j} \mathbf{w}_{j q-1}^{\kappa}, \mathbf{O}_{j q-1}^{\kappa}\right)- \\ -2\left(\mu_{j} \mathbf{w}_{j q}^{\kappa}, \mathbf{O}_{j} \mathbf{w}_{j q}^{\kappa}\right)\end{array}\right\} \leq$

$\frac{\Delta x \Delta y}{\Delta t} \sum_{q=-\infty}^{+\infty} \sum_{j=0}^{J-1}\left[\left(\mu_{j} \mathbf{O}_{j} \mathbf{w}_{j q}^{\kappa}, \mathbf{O}_{j} \mathbf{w}_{j q}^{\kappa}\right)+\right.$

$\left.+\left(\mu_{j} \mathbf{w}_{j q}^{\kappa}, \mathbf{O}_{j} \mathbf{w}_{j q}^{\kappa}\right)-\left(\mu_{j} \mathbf{O}_{j} \mathbf{w}_{j q}^{\kappa}, \mathbf{O}_{j} \mathbf{w}_{j q}^{\kappa}\right)\right]$

$\frac{\Delta x \Delta y}{\Delta t} \sum_{q=-\infty}^{+\infty} \sum_{j=0}^{J-1}\left\{\begin{array}{l}\left\{\mu_{j} \mathbf{w}_{j q-1}^{\kappa}, \mathbf{O}_{j} \mathbf{w}_{j q-1}^{\kappa}\right\}- \\ -\left(\mu_{j} \mathbf{O}_{j} \mathbf{w}_{j q-1}^{\kappa}, \mathbf{O}_{j} \mathbf{w}_{j q-1}^{\kappa}\right)+ \\ +\left(\mu_{j} \mathbf{O}_{j} \mathbf{w}_{j q-1}^{\kappa}, \mathbf{O}_{j} \mathbf{w}_{j q-1}^{\kappa}\right)- \\ -2\left(\mu_{j} \mathbf{w}_{j q}^{\kappa}, \mathbf{O}_{j} \mathbf{w}_{j q}^{\kappa}\right)\end{array}\right\}$

$=\frac{\Delta x \Delta y}{\Delta t} \sum_{q=-\infty}^{+\infty} \sum_{j=0}^{J-1}\left[\left(\mu_{j} \mathbf{w}_{j q-1}^{\kappa}, \mathbf{O}_{j} \mathbf{w}_{j q-1}^{\kappa}\right)-\right.$

$\left.-\left(\mu_{j} \mathbf{w}_{j q}^{\kappa}, \mathbf{O}_{j} \mathbf{w}_{j q}^{\kappa}\right)\right]$.
Since the initial functions are assumed to be zero at $|y|>$ $\frac{1}{2} Y$, then (see [1]) for $t=\Delta t$, the difference solution obtained by the described scheme will be obviously equal to zero if $|y|>\frac{1}{2} Y+\Delta y$ at $t=2 \Delta t$, if $|y|>\frac{1}{2} Y+2 \Delta y$, etc. The difference solution will be equal to zero on the last grid layer $t \equiv T$ if $|y|>\frac{1}{2} Y+\frac{T}{\Delta t} \Delta y$. Thus, the grid function $\mathbf{w}_{j q}^{\kappa}$ will be nonzero on any considered grid layer that only at a finite number of points. If $t$ is not too large, $\mathbf{w}_{j q}^{\kappa}=0$ if $|y|>Y$ . Taking into account this property of difference solutions, we have:

$$
\begin{aligned}
& \frac{L\left(\mathbf{u}^{\kappa}\right)-L\left(\mathbf{w}^{\kappa}\right)}{\Delta t} \leq \frac{\Delta x \Delta y}{\Delta t} \sum_{q=-\infty}^{+\infty} \sum_{j=0}^{J-1}\left[\left(\mu_{j} \mathbf{w}_{j q-1}^{\kappa},\right.\right. \\
& \left.\left.\mathbf{O}_{j} \mathbf{w}_{j q-1}^{\kappa}\right)-\left(\mu_{j} \mathbf{w}_{j q}^{\kappa}, \mathbf{O}_{j} \mathbf{w}_{j q}^{\kappa}\right)\right]=0 .
\end{aligned}
$$

Therefore

$$
\frac{L\left(\mathbf{u}^{\kappa}\right)-L\left(\mathbf{w}^{\kappa}\right)}{\Delta t} \leq 0 .
$$

, Lemma 2 is proved.

Lemma 3. Let the conditions of Theorem 2 be satisfied. Then the following inequality

$$
\frac{L\left(\mathbf{w}^{\kappa}\right)-L\left(\mathbf{v}^{\kappa}\right)}{\Delta t}<-\eta L\left(\mathbf{v}^{\kappa}\right)
$$

holds.

Proof. Introduce the following notation

$\mathbf{W}_{j q}^{\kappa}=\left[\begin{array}{c}\left(\mathbf{v}^{\mathrm{I}}\right)_{j-1, q}^{\kappa} \\ \left(\mathbf{v}^{\mathrm{II}}\right)_{j+1, q}^{\kappa}\end{array}\right], \quad|\mathbf{K}|_{j}=\left[\begin{array}{cc}\mathbf{K}_{j-1}^{+} & 0 \\ 0 & \mathbf{K}_{j+1}^{-}\end{array}\right]$,

$\mathbf{D}_{j}=\frac{\Delta t}{\Delta x}|\mathbf{K}|_{j}$

Taking into account these notations, using the difference scheme (10), calculate the quadratic form $\frac{L\left(\mathbf{w}^{\kappa}\right)-L\left(\mathbf{v}^{\kappa}\right)}{\Delta t}$. Then we obtain:

$$
\begin{aligned}
& \frac{L\left(\mathbf{w}^{\kappa}\right)-L\left(\mathbf{v}^{\kappa}\right)}{\Delta t}=\frac{\Delta x \Delta y}{\Delta t} \sum_{q=-\infty}^{+\infty} \sum_{j=0}^{J-1}\left[\left(\mathbf{w}_{j q}^{\kappa}, \mu_{j} \mathbf{w}_{j q}^{\kappa}\right)-\right. \\
&\left.-\left(\mathbf{v}_{j q}^{\kappa}, \mu_{j} \mathbf{v}_{j q}^{\kappa}\right)\right]==\frac{\Delta x \Delta y}{\Delta t} \sum_{q=-\infty}^{+\infty} \sum_{j=0}^{J-1}\left[\left(\left\{\mathbf{v}_{j q}^{\kappa}-\mathbf{D}_{j}\left[\mathbf{v}_{j q}^{\kappa}-\right.\right.\right.\right. \\
&\left.\left.\left.\left.-\mathbf{W}_{j q}^{\kappa}\right]\right\}, \mu_{j}\left\{\mathbf{v}_{j q}^{\kappa}-\mathbf{D}_{j}\left[\mathbf{v}_{j q}^{\kappa}-\mathbf{W}_{j q}^{\kappa}\right]\right\}\right)-\left(\mathbf{v}_{j q}^{\kappa}, \mu_{j} \mathbf{v}_{j q}^{\kappa}\right)\right]= \\
&= \frac{\Delta x \Delta y}{\Delta t} \sum_{q=-\infty}^{+\infty} \sum_{j=0}^{J-1}\left[\left(\mathbf{v}_{j q}^{\kappa}, \mu_{j} \mathbf{v}_{j q}^{\kappa}\right)-\left(\mathbf{v}_{j q}^{\kappa}, \mu_{j} \mathbf{v}_{j q}^{\kappa}\right)-\right. \\
&\left.-2\left(\mu_{j} \mathbf{v}_{j q}^{\kappa}, \mathbf{D}_{j}\left[\mathbf{v}_{j q}^{\kappa}-\mathbf{W}_{j q}^{\kappa}\right]\right)\right]+\frac{\Delta x \Delta y}{\Delta t} \times \\
& \times \sum_{q=-\infty}^{+\infty} \sum_{j=0}^{J-1}\left[\left(\mu_{j} \mathbf{D}_{j}\left[\mathbf{v}_{j q}^{\kappa}-\mathbf{W}_{j q}^{\kappa}\right], \mathbf{D}_{j}\left[\mathbf{v}_{j q}^{\kappa}-\mathbf{W}_{j q}^{\kappa}\right]\right)\right]= \\
& \quad \frac{\Delta x \Delta y}{\Delta t} \sum_{q=-\infty}^{+\infty} \sum_{j=0}^{J-1}\left[\left(\mu_{j} \mathbf{D}_{j}\left[\mathbf{v}_{j q}^{\kappa}-\mathbf{W}_{j q}^{\kappa}\right], \mathbf{D}_{j}\left[\mathbf{v}_{j q}^{\kappa}-\mathbf{W}_{j q}^{\kappa}\right]\right)-\right. \\
&\left.-2\left(\mu_{j} \mathbf{v}_{j q}^{\kappa}, \mathbf{D}_{j}\left[\mathbf{v}_{j q}^{\kappa}-\mathbf{W}_{j q}^{\kappa}\right]\right)\right] \\
&=\frac{\Delta x \Delta y}{\Delta t} \sum_{q=-\infty}^{+\infty} \sum_{j=0}^{J-1}\left[\left(\mu_{j} \mathbf{D}_{j} \mathbf{v}_{j q}^{\kappa}, \mathbf{D} \mathbf{v}_{j q}^{\kappa}\right)-2\left(\mu_{j} \mathbf{D} \mathbf{v}_{j q}^{\kappa}, \mathbf{D}_{j} \mathbf{W}_{j q}^{\kappa}\right)+\right. \\
&\left.+\left(\mu_{j} \mathbf{D}_{j} \mathbf{W}_{j q}^{\kappa}, \mathbf{D}_{j} \mathbf{W}_{j q}^{\kappa}\right)\right]+\frac{\Delta x \Delta y}{\Delta t} \times \\
& \times \sum_{q=-\infty}^{+\infty} \sum_{j=0}^{J-1}\left[-2\left(\mu_{j} \mathbf{v}_{j q}^{\kappa}, \mathbf{D}_{j} \mathbf{v}_{j q}^{\kappa}\right)+2\left(\mu_{j} \mathbf{v}_{j q}^{\kappa}, \mathbf{D}_{j} \mathbf{W}_{j q}^{\kappa}\right)\right]= \\
& \frac{\Delta x \Delta y}{\Delta t} \sum_{q=-\infty}^{+\infty} \sum_{j=0}^{J-1}\left[\left(\mu_{j} \mathbf{D}_{j} \mathbf{v}_{j q}^{\kappa}, \mathbf{D}_{j} \mathbf{v}_{j q}^{\kappa}\right)+\right. \\
&\left.+2\left(\mu_{j}\left(\mathbf{E}-\mathbf{D}_{j}\right) \mathbf{v}_{j q}^{\kappa}, \mathbf{D}_{j} \mathbf{W}_{j q}^{\kappa}\right)\right]+ \\
&+\frac{\Delta x \Delta y}{\Delta t} \sum_{q=-\infty}^{+\infty} \sum_{j=0}^{J-1}\left[\left(\mu_{j} \mathbf{D}_{j} \mathbf{W}_{j q}^{\kappa}, \mathbf{D}_{j} \mathbf{W}_{j q}^{\kappa}\right)-2\left(\mu_{j} \mathbf{v}_{j q}^{\kappa}, \mathbf{D}_{j} \mathbf{v}_{j q}^{\kappa}\right)\right]
\end{aligned}
$$


By virtue of the CFL condition, matrices $\left(\mathbf{E}-\mathbf{D}_{j}\right) \geq 0$ and $\left(\mathbf{E}-\mathbf{D}_{j}\right) \mu_{j} \mathbf{D}_{j}$ are positive definite diagonal matrices. Hence, we have
$2\left(\mu_{j}\left(\mathbf{E}-\mathbf{D}_{j}\right) \mathbf{v}_{j q}^{\kappa}, \mathbf{D}_{j} \mathbf{W}_{j q}^{\kappa}\right) \leq\left(\mu_{j}\left(\mathbf{E}-\mathbf{D}_{j}\right) \mathbf{v}_{j q}^{\kappa}, \mathbf{D}_{j} \mathbf{v}_{j q}^{\kappa}\right)+=e^{-\nu \Delta x} \Delta y \sum_{q=-\infty}^{+\infty} \sum_{j=0}^{J-1}\left(\left(\left(\mathbf{v}^{\mathrm{I}}\right)_{j-1, q}^{\kappa}, \mu^{+} e^{-\nu x_{j-1} \times}\right.\right.$ $+\left(\mu_{j}\left(\mathbf{E}-\mathbf{D}_{j}\right) \mathbf{W}_{j q}^{\kappa}, \mathbf{D}_{j} \mathbf{W}_{j q}^{\kappa}\right)=\left(\mu_{j} \mathbf{v}_{j q}^{\kappa}, \mathbf{D}_{j} \mathbf{v}_{j q}^{\kappa}\right)-$ $\left.\left.-\left(\mu_{j} \mathbf{D}_{j} \mathbf{v}_{j q}^{\kappa}, \mathbf{D}_{j} \mathbf{v}_{j q}^{\kappa}\right)+\left(\mu_{j} \mathbf{W}_{j q}^{\kappa}, \mathbf{D}_{j} \mathbf{W}_{j q}^{\kappa}\right)-\left(\mu_{j} \mathbf{D} \mathbf{W}_{j q}^{\kappa}, \mathbf{D} \mathbf{W}_{j q}^{\kappa}\right), \mathbf{K}_{j-1}^{+}\left(\mathbf{v}^{\mathrm{I}}\right)_{j-1, q}^{\kappa}\right)\right)=$ Taking into account this inequality, we obtain $=e^{-\nu \Delta x} \Delta y \sum_{q=-\infty}^{+\infty} \sum_{j=0}^{J-1}\left(\left(\mathbf{v}^{\mathrm{II}}\right)_{j+1, q}^{\kappa}, \mu^{-} e^{\nu x_{j+1} \times}\right.$
$\frac{L\left(\mathbf{w}^{\kappa}\right)-L\left(\mathbf{v}^{\kappa}\right)}{\Delta t}=$

$=\frac{\Delta x \Delta y}{\Delta t} \sum_{q=-\infty}^{+\infty} \sum_{j=0}^{J-1}\left[\left(\mu_{j} \mathbf{D}_{j} \mathbf{v}_{j q}^{\kappa}, \mathbf{D}_{j} \mathbf{v}_{j q}^{\kappa}\right)+\right.$

$\left.+2\left(\mu_{j}\left(\mathbf{E}-\mathbf{D}_{j}\right) \mathbf{v}_{j q}^{\kappa}, \mathbf{D}_{j} \mathbf{W}_{j q}^{\kappa}\right)\right]+$

$\left.\times \mathbf{K}_{j+1}^{-}\left(\mathbf{v}^{\mathrm{II}}\right)_{j+1, q}^{\kappa}\right)=$

$+\frac{\Delta x \Delta y}{\Delta t} \sum_{q=-\infty}^{+\infty} \sum_{j=0}^{J-1}\left[\left(\mu_{j} \mathbf{D}_{j} \mathbf{W}_{j q}^{\kappa}, \mathbf{D}_{j} \mathbf{W}_{j q}^{\kappa}\right)-\right.$

$e^{-\nu \Delta x} \Delta y \sum_{q=-\infty}^{+\infty} \sum_{j=0}^{J-1}\left(\left(\mathbf{v}^{\mathrm{I}}\right)_{j q}^{\kappa}, \mu^{+} e^{-\nu x_{j}} \mathbf{K}_{j}^{+}\left(\mathbf{v}^{\mathrm{I}}\right)_{j q}^{\kappa}\right)$

$\left.-2\left(\mu_{j} \mathbf{v}_{j q}^{\kappa}, \mathbf{D}_{j} \mathbf{v}_{j q}^{\kappa}\right)\right] \leq \frac{\Delta x \Delta y}{\Delta t} \sum_{q=-\infty}^{+\infty} \sum_{j=0}^{J-1}\left[\left(\mu_{j} \mathbf{D}_{j} \mathbf{v}_{j q}^{\kappa}, \mathbf{D}_{j} \mathbf{v}_{j q}^{\kappa}\right)+\left(\left(\mathbf{v}^{\mathrm{I}}\right)_{J-1, q}^{\kappa}, \mu^{+} e^{-\nu x_{J-1}} \mathbf{K}_{J-1}^{+}\left(\mathbf{v}^{\mathrm{I}}\right)_{J-1, q}^{\kappa}\right)\right.$

$\left.+\left(\mu_{j} \mathbf{v}_{j q}^{\kappa}, \mathbf{D}_{j} \mathbf{v}_{j q}^{\kappa}\right)-\left(\mu_{j} \mathbf{D}_{j} \mathbf{v}_{j q}^{\kappa}, \mathbf{D}_{j} \mathbf{v}_{j q}^{\kappa}\right)\right]+$

$e^{-\nu \Delta x} \Delta y \sum_{q=-\infty}^{+\infty} \sum_{j=0}^{J-1}\left(\left(\mathbf{v}^{\mathrm{II}}\right)_{j q}^{\kappa}, \mu^{-} e^{\nu x_{j}} \mathbf{K}_{j}^{-}\left(\mathbf{v}^{\mathrm{II}}\right)_{j q}^{\kappa}\right)$

$\frac{\Delta x \Delta y}{\Delta t} \sum_{q=-\infty}^{+\infty} \sum_{j=0}^{J-1}\left[\left(\mu_{j} \mathbf{W}_{j q}^{\kappa}, \mathbf{D}_{j} \mathbf{W}_{j q}^{\kappa}\right)-\left(\mu_{j} \mathbf{D}_{j} \mathbf{W}_{j q}^{\kappa}, \mathbf{D}_{j} \mathbf{W}_{j q}^{\kappa}\right)\right]+e^{-\nu \Delta x} \Delta y \sum_{q=-\infty}^{+\infty}-\left(\left(\mathbf{v}^{\mathrm{II}}\right)_{0 q}^{\kappa}, \mu^{-} e^{\nu x_{0}} \mathbf{K}_{0}^{-}\left(\mathbf{v}^{\mathrm{II}}\right)_{0 q}^{\kappa}\right)+$

$\frac{\Delta x \Delta y}{\Delta t} \sum_{q=-\infty}^{+\infty} \sum_{j=0}^{J-1}\left[\left(\mu_{j} \mathbf{D}_{j} \mathbf{W}_{j q}^{\kappa}, \mathbf{D}_{j} \mathbf{W}_{j q}^{\kappa}\right)-2\left(\mu_{j} \mathbf{v}_{j q}^{\kappa}, \mathbf{D}_{j} \mathbf{v}_{j q}^{\kappa}\right)\right]$

$=\frac{\Delta x \Delta y}{\Delta t} \sum_{q=-\infty}^{+\infty} \sum_{j=0}^{J-1}\left[\left(\mu_{j} \mathbf{W}_{j q}^{\kappa}, \mathbf{D}_{j} \mathbf{W}_{j q}^{\kappa}\right)-\left(\mu_{j} \mathbf{v}_{j q}^{\kappa}, \mathbf{D}_{j} \mathbf{v}_{j q}^{\kappa}\right)\right]$

or $\frac{L\left(\mathbf{w}^{\kappa}\right)-L\left(\mathbf{v}^{\kappa}\right)}{\Delta t} \leq \frac{\Delta x \Delta y}{\Delta t} \sum_{q=-\infty}^{+\infty} \sum_{j=0}^{J-1}\left[\left(\mu_{j} \mathbf{W}_{j q}^{\kappa}, \mathbf{D}_{j} \mathbf{W}_{j q}^{\kappa}\right)-\right.$

$\left.-\left(\mu_{j} \mathbf{v}_{j q}^{\kappa}, \mathbf{D}_{j} \mathbf{v}_{j q}^{\kappa}\right)\right]$.

$\left(\left(\mathbf{v}^{\mathrm{II}}\right)_{J q}^{\kappa}, \mu^{-} e^{\nu x_{J}} \mathbf{K}_{J}^{-}\left(\mathbf{v}^{\mathrm{II}}\right)_{J q}^{\kappa}\right)=$

$e^{-\nu \Delta x} \Delta y \sum_{q=-\infty}^{+\infty} \sum_{j=0}^{J-1}\left(\mathbf{v}_{j q}^{\kappa}, \mu_{j} \mathbf{K}_{j} \mathbf{v}_{j q}^{\kappa}\right)+$

$+e^{-\nu \Delta x} \Delta y \sum_{q=-\infty}^{+\infty}\left(\left(\mathbf{v}^{\mathrm{I}}\right)_{-1, q}^{\kappa}, \mu^{+} e^{-\nu x_{0}} \mathbf{K}_{-1}^{+}\left(\mathbf{v}^{\mathrm{I}}\right)_{-1, q}^{\kappa}\right)-$

$\left(\left(\mathbf{v}^{\mathrm{I}}\right)_{J-1, q}^{\kappa}, \mu^{+} e^{-\nu x_{J}} \mathbf{K}_{J-1}^{+}\left(\mathbf{v}^{\mathrm{I}}\right)_{J-1, q}^{\kappa}\right)$

$-e^{-\nu \Delta x} \Delta y \sum_{q=-\infty}^{+\infty}\left(\left(\mathbf{v}^{\mathrm{II}}\right)_{0 q}^{\kappa}, \mu^{-} e^{\nu x_{0}} \mathbf{K}_{0}^{-}\left(\mathbf{v}^{\mathrm{II}}\right)_{0 q}^{\kappa}\right)+$

$\left(\left(\mathbf{v}^{\mathrm{II}}\right)_{J q}^{\kappa}, \mu^{-} e^{\nu x_{J}} \mathbf{K}_{J}^{-}\left(\mathbf{v}^{\mathrm{II}}\right)_{J q}^{\kappa}\right)=$

$=e^{-\nu \Delta x} \Delta y \sum_{q=-\infty}^{+\infty} \sum_{j=0}^{J-1}\left(\mathbf{v}_{j q}^{\kappa}, \mu_{j} \mathbf{K}_{j} \mathbf{v}_{j q}^{\kappa}\right)+$

$+e^{-\nu \Delta x} \Delta y \sum_{q=-\infty}^{+\infty}\left(\left[\begin{array}{c}\left(\mathbf{v}^{\mathrm{I}}\right)_{-1, q}^{\kappa} \\ \left(\mathbf{v}^{\mathrm{II}}\right)_{J q}^{\kappa}\end{array}\right]\right.$,

Transform separately the first quadratic form in the right side of this inequality:

$$
\begin{aligned}
& \left.\left[\begin{array}{cc}
\mu^{+} e^{-\nu x_{0}} \mathbf{K}_{-1}^{+} & 0 \\
0 & \mu^{-} e^{\nu x_{J-1}} \mathbf{K}_{J}^{-}
\end{array}\right]\left[\begin{array}{c}
\left(\mathbf{v}^{\mathrm{I}}\right)_{-1, q}^{\kappa} \\
\left(\mathbf{v}^{\mathrm{II}}\right)_{J q}^{\kappa}
\end{array}\right]\right)- \\
& -e^{-\nu \Delta x} \Delta y \sum_{q=-\infty}^{+\infty}\left(\left[\begin{array}{c}
\left(\mathbf{v}^{\mathrm{I}}\right)_{J-1, q}^{\kappa} \\
\left(\mathbf{v}^{\mathrm{II}}\right)_{0 q}^{\kappa}
\end{array}\right],\right. \\
& \left.\times\left[\begin{array}{cc}
\mu^{+} e^{-\nu x_{J}} \mathbf{K}_{J-1}^{+} & 0 \\
0 & \mu^{-} e^{\nu x_{-1}} \mathbf{K}_{0}^{-}
\end{array}\right]\left[\begin{array}{c}
\left(\mathbf{v}^{\mathrm{I}}\right)_{J-1, q}^{\kappa} \\
\left(\mathbf{v}^{\mathrm{II}}\right)_{0 q}^{\kappa}
\end{array}\right]\right)
\end{aligned}
$$

Here

$$
\begin{aligned}
& \frac{\Delta x \Delta y}{\Delta t} \sum_{q=-\infty}^{+\infty} \sum_{j=0}^{J-1}\left(\mu_{j} \mathbf{W}_{j q}^{\kappa}, \mathbf{D}_{j} \mathbf{W}_{j q}^{\kappa}\right)= \\
& =\Delta y \sum_{q=-\infty}^{+\infty} \sum_{j=0}^{J-1}\left(\mathbf{W}_{j q}^{\kappa},\right. \\
& \left.\left[\begin{array}{cc}
\mu^{+} e^{-\nu x_{j}} \mathbf{K}_{j-1}^{+} & 0 \\
0 & \mu^{-} e^{\nu x_{j}} \mathbf{K}_{j+1}^{-}
\end{array}\right] \mathbf{W}_{j q}^{\kappa}\right)= \\
& e^{-\nu \Delta x} \Delta y \sum_{q=-\infty}^{+\infty} \sum_{j=0}^{J-1}\left(\mathbf{W}_{j q}^{\kappa},\right. \\
& \left.\left[\begin{array}{cc}
\mu^{+} e^{-\nu x_{j-1}} \mathbf{K}_{j-1}^{+} & 0 \\
0 & \mu^{-} e^{\nu x_{j+1}} \mathbf{K}_{j+1}^{-}
\end{array}\right] \mathbf{W}_{j q}^{\kappa}\right)=
\end{aligned}
$$$$
|\mathbf{K}|_{j}=\left[\begin{array}{cc}
\mathbf{K}_{j}^{+} & 0 \\
0 & \mathbf{K}_{j}^{-}
\end{array}\right] .
$$

Taking into account the boundary conditions

$$
\left(\begin{array}{c}
\left(\mathbf{v}^{\mathrm{I}}\right)_{-1, q}^{\kappa+1} \\
\left(\mathbf{v}^{\mathrm{II}}\right)_{J q}^{\kappa+1}
\end{array}\right)=\left(\begin{array}{cc}
0 & \mathbf{s} \\
\mathbf{r} & 0
\end{array}\right)\left(\begin{array}{c}
\left(\mathbf{v}^{\mathrm{I}}\right)_{J-1, q}^{\kappa+1} \\
\left(\mathbf{v}^{\mathrm{II}}\right)_{0 q}^{\kappa+1}
\end{array}\right)
$$

we have 


$$
\begin{aligned}
& \frac{\Delta x \Delta y}{\Delta t} \sum_{q=-\infty}^{+\infty} \sum_{j=0}^{J-1}\left(\mu_{j} \mathbf{W}_{j q}^{\kappa}, \mathbf{D}_{j} \mathbf{W}_{j q}^{\kappa}\right)=e^{-\nu \Delta x} \Delta y \times \\
& \times \sum_{q=-\infty}^{+\infty} \sum_{j=0}^{J-1}\left(\mathbf{v}_{j q}^{\kappa}, \mu_{j} \mathbf{K}_{j} \mathbf{v}_{j q}^{\kappa}\right)+\Delta y \sum_{q=-\infty}^{+\infty}\left(\begin{array}{cc}
0 & \mathbf{s} \\
\mathbf{r} & 0
\end{array}\right) \times \\
& \times\left(\begin{array}{c}
\left(\mathbf{v}^{\mathrm{I}}\right)_{J-1, q}^{\kappa+1} \\
\left(\mathbf{v}^{\mathrm{II}}\right)_{0 q}^{\kappa+1}
\end{array}\right),\left[\begin{array}{cc}
\mu^{+} e^{-\nu x_{0}} \mathbf{K}_{-1}^{+} & 0 \\
0 & \mu^{-} e^{\nu x_{J-1}} \mathbf{K}_{J}^{-}
\end{array}\right] \times \\
& \times\left(\begin{array}{ll}
0 & \mathbf{s} \\
\mathbf{r} & 0
\end{array}\right)\left(\begin{array}{c}
\left(\mathbf{v}^{\mathrm{I}}\right)_{J-1, q}^{\kappa+1} \\
\left(\mathbf{v}^{\mathrm{II}}\right)_{0 q}^{\kappa+1}
\end{array}\right)- \\
& -\Delta y \sum_{q=-\infty}^{+\infty}\left(\left[\begin{array}{c}
\left(\mathbf{v}^{\mathrm{I}}\right)_{J-1, q}^{\kappa} \\
\left(\mathbf{v}^{\mathrm{II}}\right)_{0 q}^{\kappa}
\end{array}\right]\right. \\
& \left.\times\left[\begin{array}{cc}
\mu^{+} e^{-\nu x_{J}} \mathbf{K}_{J-1}^{+} & 0 \\
0 & \mu^{-} e^{\nu x_{-1}} \mathbf{K}_{0}^{-}
\end{array}\right]\left[\begin{array}{c}
\left(\mathbf{v}^{\mathrm{I}}\right)_{J-1, q}^{\kappa} \\
\left(\mathbf{v}^{\mathrm{II}}\right)_{0 q}^{\kappa}
\end{array}\right]\right) \\
& \text { Suppose that the dissipativity condition } \\
& {\left[\begin{array}{cc}
\mu^{+} e^{-\nu x_{J}} \mathbf{K}_{J-1}^{+} & 0 \\
0 & \mu^{-} e^{\nu x_{-1}} \mathbf{K}_{0}^{-}
\end{array}\right]-\left(\begin{array}{cc}
0 & \mathbf{r} \\
\mathbf{s} & 0
\end{array}\right) \times} \\
& \times\left[\begin{array}{cc}
\mu^{+} e^{-\nu x_{0}} \mathbf{K}_{-1}^{+} & 0 \\
0 & \mu^{-} e^{\nu x_{J-1}} \mathbf{K}_{J}^{-}
\end{array}\right]\left(\begin{array}{cc}
0 & \mathbf{s} \\
\mathbf{r} & 0
\end{array}\right)>0 \text { holds. }
\end{aligned}
$$

Then we obtain

$$
\frac{\Delta x \Delta y}{\Delta t} \sum_{q=-\infty}^{+\infty} \sum_{j=0}^{J-1}\left(\mu_{j} \mathbf{W}_{j q}^{\kappa}, \mathbf{D}_{j} \mathbf{W}_{j q}^{\kappa}\right) \leq
$$$$
\leq e^{-\nu \Delta x} \Delta y \sum_{q=-\infty}^{+\infty} \sum_{j=0}^{J-1}\left(\mathbf{v}_{j q}^{\kappa}, \mu_{j} \mathbf{K}_{j} \mathbf{v}_{j q}^{\kappa}\right) \text { and, hence, }
$$$$
\frac{L\left(\mathbf{w}^{\kappa}\right)-L\left(\mathbf{v}^{\kappa}\right)}{\Delta t} e^{-\nu \Delta x} \Delta y \sum_{q=-\infty}^{+\infty} \sum_{j=0}^{J-1}\left(\mathbf{v}_{j q}^{\kappa}, \mu_{j} \mathrm{~K}_{j} \mathbf{v}_{j q}^{\kappa}\right)-
$$$$
-\Delta y \sum_{q=-\infty}^{+\infty} \sum_{j=0}^{J-1}\left(\mathbf{v}_{j q}^{\kappa}, \mu_{j}|\mathbf{K}|_{j} \mathbf{v}_{j q}^{\kappa}\right)=
$$$$
=\Delta y \sum_{q=-\infty}^{+\infty} \sum_{j=0}^{J-1}\left(\mathbf{v}_{j q}^{\kappa}, \mu_{j}\left[e^{-\nu \Delta x} \mathrm{~K}_{j}-|\mathbf{K}|_{j}\right] \mathbf{v}_{j q}^{\kappa}\right)=
$$$$
=\Delta y \sum_{q=-\infty}^{+\infty} \sum_{j=0}^{J-1}\left(\mathbf{v}_{j q}^{\kappa}, \mu_{j}\left[e^{-\nu \Delta x} \mathrm{~K}_{j}-\mathrm{K}_{j}+\mathrm{K}_{j}-|\mathbf{K}|_{j}\right] \mathbf{v}_{j q}^{\kappa}\right)=
$$$$
=\Delta y \sum_{q=-\infty}^{+\infty} \sum_{j=0}^{J-1}\left(\mathbf{v}_{j q}^{\kappa}, \mu_{j}\left[e^{-\nu \Delta x} \mathrm{~K}_{j}-\mathrm{K}_{j}\right] \mathbf{v}_{j q}^{\kappa}\right)+
$$$$
+\Delta y \sum_{q=-\infty}^{+\infty} \sum_{j=0}^{J-1}\left(\mathbf{v}_{j q}^{\kappa}, \mu_{j}\left[\mathrm{~K}_{j}-|\mathbf{K}|_{j}\right] \mathbf{v}_{j q}^{\kappa}\right)=
$$$$
=\left(e^{-\nu \Delta x}-1\right) \Delta y \sum_{q=-\infty}^{+\infty} \sum_{j=0}^{J-1}\left(\mathbf{v}_{j q}^{\kappa}, \mu_{j} \mathrm{~K}_{j} \mathbf{v}_{j q}^{\kappa}\right)+
$$

$\Delta y \sum_{q=-\infty}^{+\infty} \sum_{j=0}^{J-1}\left(\mathbf{v}_{j q}^{\kappa}, \mu_{j}\left[\begin{array}{cc}\mathbf{K}_{j}^{+}-\mathbf{K}_{j-1}^{+} & 0 \\ 0 & \mathbf{K}_{j}^{-}-\mathbf{K}_{j+1}^{-}\end{array}\right] \mathbf{v}_{j q}^{\kappa}\right)=$

$$
=\quad-\nu \Delta x \Delta y \sum_{q=-\infty}^{+\infty} \sum_{j=0}^{J-1}\left(\mathbf{v}_{j q}^{\kappa}, \mu_{j} \mathrm{~K}_{j} \mathbf{v}_{j q}^{\kappa}\right) \quad+
$$$$
\Delta x \Delta y \sum_{q=-\infty}^{+\infty} \sum_{j=0}^{J-1}\left(\mathbf{v}_{j q}^{\kappa}, \mu_{j} \mathrm{~K}^{\prime}{ }_{j} \mathbf{v}_{j q}^{\kappa}\right) \text {. }
$$

Since according to the condition of Theorem 2, there are constants

$$
\alpha=\min _{\substack{1 \leq i \leq n \\ 0 \leq j \leq J-1}}\left|k_{i, j}\right|, \beta=\max _{\substack{1 \leq i \leq n \\ 0 \leq j \leq J-1}}\left|k_{i, j}^{\prime}\right|
$$

such that $0<\nu \alpha e^{-\nu \Delta x}-\beta<1$, we get

$$
\frac{L\left(\mathbf{w}^{\kappa}\right)-L\left(\mathbf{v}^{\kappa}\right)}{\Delta t}<-\eta L\left(\mathbf{v}^{\kappa}\right) \text {. }
$$

Here

$$
\eta=\nu \alpha e^{-\nu \Delta x}-\beta
$$

Lemma 3 is proved.

As a result, lemmas 1-3 imply the following inequalities:

$\frac{L\left(\mathbf{v}^{\kappa+1}\right)-L\left(\mathbf{u}^{\kappa}\right)}{\Delta t} \leq 0, \quad \frac{L\left(\mathbf{u}^{\kappa}\right)-L\left(\mathbf{w}^{\kappa}\right)}{\Delta t} \leq 0$,

Summing up these inequalities, we have the following for the quadratic form

$\frac{L\left(\mathbf{v}^{\kappa+1}\right)-L\left(\mathbf{v}^{\kappa}\right)}{\Delta t}: \frac{L\left(\mathbf{v}^{\kappa+1}\right)-L\left(\mathbf{v}^{\kappa}\right)}{\Delta t}=\frac{L\left(\mathbf{v}^{\kappa+1}\right)-L\left(\mathbf{u}^{\kappa}\right)}{\Delta t}+$
$+\frac{L\left(\mathbf{u}^{\kappa}\right)-L\left(\mathbf{w}^{\kappa}\right)}{\Delta t}+\frac{L\left(\mathbf{w}^{\kappa}\right)-L\left(\mathbf{v}^{\kappa}\right)}{\Delta t}<-\eta L\left(\mathbf{v}^{\kappa}\right)$ or $\frac{L^{\kappa+1}-L^{\kappa}}{\Delta t}<$ $-\eta L^{\kappa}$ or $\frac{L^{\kappa+1}-L^{\kappa}}{\Delta t}<-\eta L^{\kappa}$. Recursively applying this inequality, we obtain

$L^{\kappa+1}<(1-\Delta t \eta)^{\kappa+1} L^{0} \leq e^{-\eta \Delta t(\kappa+1)} L^{0}=$ $e^{-\eta t_{\kappa+1}} L^{0}, \kappa=0, \ldots, N-1$.

Denote

$C_{1}=\min _{\substack{1 \leq i \leq n \\ 0 \leq j \leq J-1}}\left\{\varpi_{i j}:\left|\mu_{j}-\varpi_{i j} \mathbf{E}\right|=0\right\}, \quad C_{2}=$ $\max _{\substack{1 \leq i \leq n \\ 0 \leq j \leq J-1}}\left\{\varpi_{i j}:\left|\mu_{j}-\varpi_{i j} \mathbf{E}\right|=0\right\}$,

$0 \leq j \leq J-1$
Then

$$
C_{1} \mathbf{E} \leq \mu_{j} \leq C_{2} \mathbf{E}, \quad j=0, \ldots, J-1
$$

It follows from here that

$$
\begin{aligned}
& C_{1} \Delta x \Delta y \sum_{q=-\infty}^{+\infty} \sum_{j=0}^{J-1}\left(\mathbf{v}_{j q}^{\kappa}, \mathbf{v}_{j q}^{\kappa}\right) \leq L^{\kappa} \leq \\
\leq & C_{2} e^{-\eta t_{\kappa}} \Delta x \Delta y \sum_{q=-\infty}^{+\infty} \sum_{j=0}^{J-1}\left(\mathbf{v}_{j q}^{0}, \mathbf{v}_{j q}^{0}\right), \quad \kappa=0, \ldots, N, \\
\Delta & x \Delta y \sum_{q=-\infty}^{+\infty} \sum_{j=0}^{J-1}\left(\mathbf{v}_{j q}^{\kappa}, \mathbf{v}_{j q}^{\kappa}\right) \leq C e^{-\eta t_{\kappa}} \Delta x \Delta y \times \\
= & \sum_{q=-\infty}^{+\infty} \sum_{j=0}^{J-1}\left(\mathbf{v}_{j q}^{0}, \mathbf{v}_{j q}^{0}\right), \quad \kappa=0, \ldots, N ; C=C_{2} / C_{1} .
\end{aligned}
$$

Hence, the numerical solution $\mathbf{v}_{j q}^{n}$ of the mixed problem is exponentially stable in the $\mathbf{L}_{2}$-norm.

Theorem 2 is proved.

\section{Conclusions}

In conclusion, we note that the stability of a difference splitting scheme was studied in the work for the numerical calculation of stable solutions of a two-dimensional linear hyperbolic system with dissipative boundary conditions in the case of variable coefficients with lower terms. A discrete analogue of the Lyapunov function is constructed for the numerical value of stable solutions of a two-dimensional linear hyperbolic system with dissipative boundary conditions in the case of variable coefficients with lower terms. An a priori estimate is obtained for the discrete analogue of the Lyapunov function. The obtained prior estimate allows us to assert the exponential stability of the numerical solution. A theorem on the exponential stability of the solution of both the differential problem and the difference split-ting scheme for linear hyperbolic systems in the corresponding norms is proved. Consequently, this gives us the opportunity to prove the convergence of a stable numerical solution to a stable solution of a differential problem. 


\section{Acknowledgements}

This work was supported by Universiti Sains Islam Malaysia (USIM) under RMC Research Grant Scheme (FRGS, 2018). Project code is USIM/FRGS/FST/055002/51118

\section{REFERENCES}

[1] Godunov S.K. (1979) Equations of mathematical physics.(in Russian) M.Nauka, 372 p.

[2] Bastin G., and Coron J.M. (2016) Stability and Boundary Stabilization of 1-D Hyperbolic Systems. Progress in Nonlinear Differential Equations and Their Applications, Vol 88, Birkhäuser Basel. 2016.

[3] Blokhin A.M., Aloev R.D. (1993) Energy integrals and their applications to the study of the stability of the difference schemes.(in Russian) Novosibirsk State University Press, 1993,224 p.

[4] Aloev R.D., Eshkuvatov Z.K., Davlatov Sh.O., Nik Long NMA (2014) Sufficient condition of stability of finite element method for symmetric t-hyperbolic systems with constant coefficients. Computers and Mathematics with Applications, 68,2014,pp 1194-1204.

[5] Aloev R.D., Blokhin A.M., Hudayberganov M.U. (2014) One Class of Stable Difference Schemes for Hyperbolic System. American Journal of Numerical Analysis. 2(3),2014, pp 85-89.

[6] Aloev R.D., Davlatov Sh.O., Eshkuvatov Z.K., Nik Long NMA (2016) Uniqueness solution of the finite elements scheme for symmetric hyperbolic systems with variable coefficients. Malaysian Journal of Mathematical Sciences (MJMS), 10(S),2016,pp 49-60.

[7] Aloev R.D., Khudoyberganov M.U., Blokhin A.M. (2018) Construction and research of adequate computational models for quasilinear hyperbolic systems. NUMERICAL ALGEBRA, CONTROL AND OPTIMIZATION. Volume 8, Number 3, September 2018 pp 287-299.

[8] Aloev R.D., Eshkuvatov Z.K., Khudayberganov M.U., Nik Long NMA (2018) A discrete analogue of energy integral for a difference scheme for quasilinear hyperbolic systems. Applied Mathematics, 2018, 9, pp 789-805.

[9] Aloev R.D., Khudoyberganov M.U., Nematova D.E. (2018) On stability of the difference splitting scheme for hyperbolic systems.Uzbek Mathematical Journal, Number 2,2018,pp 4-16.

[10] Aloev R.D., Khudoyberganov M.U., (2018) A discrete analogue of the Lyapunov function for hyperbolic systems. Contemporary Mathematics. Fundamental Directions, mathnet.ru/rus 2018, Volume 64, Number 4, pp 591-602.doi.org/10.22363/24133639-2018-64-4-591-602 